\section{(6) OPEN ACCESS}

\title{
Proton pump inhibitors alter the composition of the gut microbiota
}

\author{
Matthew A Jackson, ${ }^{1}$ Julia K Goodrich, ${ }^{2,3}$ Maria-Emanuela Maxan, ${ }^{4}$ \\ Daniel E Freedberg, ${ }^{5}$ Julian A Abrams, ${ }^{5}$ Angela C Poole, ${ }^{2,3}$ Jessica L Sutter, ${ }^{2,3}$ \\ Daphne Welter, ${ }^{2,3}$ Ruth E Ley, ${ }^{2,3}$ Jordana T Bell, ${ }^{1}$ Tim D Spector, ${ }^{1}$ Claire J Steves $^{1}$
}

- Additional material is published online only. To view please visit the journal online (http://dx.doi.org/10.1136/ gutjnl-2015-310861)

${ }^{1}$ Department of Twin Research and Genetic Epidemiology, King's College London, London, UK

${ }^{2}$ Department of Molecular Biology and Genetics, Cornell University, Ithaca, New York, USA

${ }^{3}$ Department of Microbiology, Cornell University, Ithaca, New York, USA

${ }^{4}$ Clinical Age Research Unit, Kings College Hospital

Foundation Trust, London, UK ${ }^{5}$ Division of Digestive and Liver Diseases, Department of Medicine, Columbia University Medical Center, New York, New York, USA

\section{Correspondence to} Dr Claire J Steves, Department of Twin Research \& Genetic Epidemiology, King's College London, St Thomas' Hospital Campus, 3rd \& 4th Floor South Wing Block D, Westminster Bridge Road, London SE1 7EH, UK; claire.j. steves@kcl.ac.uk

Received 6 October 2015 Revised 9 November 2015 Accepted 25 November 2015 Published Online First 30 December 2015

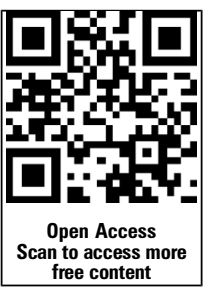

\section{CrossMark}

$$
\begin{aligned}
& \text { To cite: Jackson MA, } \\
& \text { Goodrich JK, Maxan M-E, } \\
& \text { et al. Gut 2016:65:749- }
\end{aligned}
$$
756.

\section{ABSTRACT}

Objective Proton pump inhibitors (PPIs) are drugs used to suppress gastric acid production and treat $\mathrm{Gl}$ disorders such as peptic ulcers and gastro-oesophageal reflux. They have been considered low risk, have been widely adopted, and are often over-prescribed. Recent studies have identified an increased risk of enteric and other infections with their use. Small studies have identified possible associations between PPI use and GI microbiota, but this has yet to be carried out on a large population-based cohort.

Design We investigated the association between PPI usage and the gut microbiome using 165 ribosomal RNA amplification from faecal samples of 1827 healthy twins, replicating results within unpublished data from an interventional study.

Results We identified a significantly lower abundance in gut commensals and lower microbial diversity in PPI users, with an associated significant increase in the abundance of oral and upper GI tract commensals. In particular, significant increases were observed in Streptococcaceae. These associations were replicated in an independent interventional study and in a paired analysis between 70 monozygotic twin pairs who were discordant for PPI use. We propose that the observed changes result from the removal of the low $\mathrm{pH}$ barrier between upper $\mathrm{Gl}$ tract bacteria and the lower gut. Conclusions Our findings describe a significant impact of PPIs on the gut microbiome and should caution overuse of PPIs, and warrant further investigation into the mechanisms and their clinical consequences.

\section{INTRODUCTION}

Proton pump inhibitors (PPIs) are used to increase gastric $\mathrm{pH}$ by suppressing acid production. They are pro-drugs, only becoming functional in the acidic environment of the stomach. Here, activated PPIs inhibit hydrogen-potassium pumps $(\mathrm{H}+/ \mathrm{K}+$ ATPases), transmembrane proteins responsible for releasing hydrochloric acid into the lumen of the stomach. PPIs inhibit $\mathrm{H}+/ \mathrm{K}+$ ATPases by binding covalently to the transmembrane domain, with return of acid production dependent on the turnover of new $\mathrm{H}+/ \mathrm{K}+$ ATPases once PPIs have left the system. ${ }^{1}$

PPIs are frequently used to treat GI tract disorders such as bleeding peptic ulcers, erosive esophagitis, and gastroesophageal reflux. ${ }^{2-4}$ They are also used prophylactically to prevent stress ulcers and to

\section{Significance of this study}

What is already known on this subject?

- Proton pump inhibitors (PPIs) are widely, and often over, used but recently have been associated with a number of side effects, including an increased risk of Clostridium difficile infection.

- The increased risk of infection may be mediated by alterations to the gut microbiota, as observed with antibiotics.

- Previous studies have demonstrated associations between PPI use and the gut microbiota, but have been limited in size.

\section{What are the new findings?}

- In a large healthy twin cohort, we identify significant associations between the composition of the gut microbiota and PPI use.

- The most striking association is an increase in Lactobacillales, particularly Streptococcaceae, in PPI users.

- The strongest associations replicated in a small interventional dataset indicating causality.

- Finally, we show that bacterial families increasing with PPI use are more likely to be pharyngeal, not gut, commensals.

\section{How might it impact on clinical practice in} the foreseeable future?

- The observed alterations to the gut microbiota with PPI use may be responsible for the observed increases in infection risk, and therefore provide targets for research to mitigate these risks.

- The potential consequences of these changes are motivation for caution against unnecessary provision of PPIs.

reduce GI toxicity associated with certain medications, including non-steroidal anti-inflammatory drugs, aspirin, and steroids, sometimes despite a paucity of evidence. ${ }^{5-8}$ PPIs are one of the most profitable classes of drugs in the world ${ }^{9}$; however, the high cost to healthcare systems has led to investigations into possible over-utilisation. These show that over $70 \%$ of PPI prescriptions may be inappropriate, ${ }^{10-12}$ with the majority of over-utilisation 
stemming from unnecessary stress ulcer prophylaxis in patients who do not meet the evidence-based criteria, and a lack of re-assessment of PPI use in the community. ${ }^{12}$

The use of PPIs has generally been considered safe, with low reported incidences of serious adverse outcomes. ${ }^{13-15}$ However, recently a number of side effects have been identified, including nutritional deficiencies, increased risk of bone fracture, and risks of enteric and other infections ${ }^{16-19}$; notably, increased risks of community acquired pneumonia and Clostridium difficile infection where PPIs may carry a high risk equivalent to that of oral antibiotics. $^{20} 21$

The term microbiome refers to the ecology and functionality of the microbial population within an environment. Nearly every site of the human body has a distinct microbiome with bacterial composition determined by environmental and inter-microbial influences. $^{22} 23$ Using amplification and sequencing of the variable regions of the $16 \mathrm{~S}$ ribosomal subunit it is possible to profile the taxonomic composition of the microbiome of a given sample. ${ }^{23}$ Application of this technique has shown changes to gut microbiota in a range of conditions, from IBD to obesity and frailty. $^{24-26}$ Thus, factors affecting the microbiome have the potential to drive important secondary effects on health. For example, alterations to microbial communities caused by oral antibiotics may underlie their association with increased $C$ difficile infection, ${ }^{27}$ and the same could be true for PPIs.

Previous small-scale case-control studies indicate that PPI use can influence the microbiome, but have been limited by focusing on younger individuals or patients presenting a GI disorder, with some conflicting results. ${ }^{28-32}$

Here we investigate the association between PPI usage and the gut microbiota in the largest study published to date, using $16 \mathrm{~S}$ rRNA profiling of faecal samples collected from over 1800 healthy elderly twin volunteers, allowing adjustment for environmental and heritable factors influencing both PPI use and the microbiome. We identified significant alterations to the diversity and composition of the gut microbiota in PPI users, a number of which were replicated in an intervention study. We also identified a potential mechanism by which PPIs could induce such changes.

\section{METHODS}

\section{Microbiota composition analysis}

One thousand and eighty-one faecal samples from the TwinsUK cohort had been sequenced as part of a previous study; a further $\sim 1000$ twin samples were collected and processed under the same protocol producing reads with an average length of $253 \mathrm{nt}$ after barcode removal. ${ }^{33}$ Previously generated sequencing was combined with new data and quality filtering and ecological analysis performed using QIIME. ${ }^{34}$ Sequences were collapsed to operational taxonomic units (OTUs) using open reference clustering with Greengenes v13_8 at 97\% sequence similarity. The OTU table was then sub-set to samples from twins with PPI usage data for use in subsequent analyses.

\section{Medication and GI indication data}

PPI use was self-reported at multiple time points up to 10 years before and including microbiome assessment. Use was scored as positive if an individual had reported usage at any time, even if there was a more recent negative report. This method was chosen, as PPI use is often intermittent, the longevity of any PPI mediated microbiome effects are unknown, and most misclassifications would be non-users appearing as users, which would act to reduce the strength of any observed associations. Positive PPI use was recorded a median of 3 years before microbiota assessment (IQR 0.2-4.7 years).
GI indications were scored similarly based on self-reported or professionally diagnosed indications for PPI prescription. As for PPI use, multiple time points were available and individuals were considered positive if any indication had ever been reported. Positive GI indications were a median of 1.5 years from faecal sampling (IQR 0-3.8 years).

Self-reported antibiotic use within the previous month was recorded at the time of sample collection for the majority of individuals, with drug details provided where applicable. Binary scores created from these data were corrected to reflect reported treatments, removing individuals where the reported drug was not an antibiotic.

\section{Cohort and covariate data collection}

Within TwinsUK 1827 individuals had both PPI data and faecal samples. The average age was 62 years (range 19-88 years) and $90 \%$ were female. The gender and age distribution resulted from historical study recruitment within the cohort. ${ }^{35}$ Physical measurements such as height and weight were measured at the time of sample collection.

Habitual dietary patterns were represented by the first five principle components (PCs) of food frequency questionnaires (FFQs) collected before sample collection. These have previously been shown to account for the majority of habitual diet variance and correspond to dietary types (given the names of fruit and vegetable rich, high alcohol, traditional English dieting and low meat diets, respectively). ${ }^{36}$ Frailty was quantified as a Frailty Index (FI) using the proportion of 39 binary health deficits that each individual displayed (see online supplementary table S1) from the Healthy Ageing Twin Study collected in 20072010. ${ }^{35} 37$ Covariate distributions were analysed using two-tailed Wilcoxon rank sum tests to compare the distributions of PPI users and non-users, with a significance threshold of $\mathrm{p}<0.05$.

\section{$\alpha$ Diversity}

The 1827 samples had a mean OTU count of 82130 ( $s=40506$, range $10460-380500$ ). The OTU table was rarefied to a depth of 10000 sequences and used to generate Shannon, Chao1 and phylogenetic diversity indices, as well as observed OTU counts. One-tailed Wilcoxon rank sum tests were performed to test for lower diversity in PPI users versus non-users, ${ }^{32}$ taking a significance threshold of $\mathrm{p}<0.05$ on the full set of 1827 individuals.

Mixed effects models were created using the lme4 package in $\mathrm{R},{ }^{38}$ with $\alpha$ diversity metrics as the response variable to assess the ability of PPI status to predict diversity. Technical covariates included sequencing run and depth of sample sequencing. Other covariates included family, twin structure (a variable of unique values the same for monozygotic (MZ) but different for dizygotic (DZ) twins), diet (the first five PCs from FFQs), age, body mass index (BMI), FI (root normalised), and GI indication status. The Anova function was used to compare the ability of models with and without PPI status to predict each $\alpha$ diversity metric, using the subset of 1200 individuals with complete covariate data.

\section{OTU and taxonomic associations}

Mixed effects models were again used to identify associations between PPI use and OTU and taxa abundances on 1200 individuals having complete covariate data. OTUs present in $<25 \%$ of individuals were discarded and the remaining counts converted to $\log$ transformed relative abundances (with the addition of $10^{-6}$ for zero counts). OTU abundances were used as response variables with covariates as above also including the Shannon index, to reduce associations with OTU markers of $\alpha$ 
diversity. The ability of models including and not including PPI status as a covariate to predict abundance of each OTU was quantified using the Anova function in R. p Values were FDR (false discovery rate) corrected using the 'qvalue' package with a significance threshold of $5 \% .{ }^{39}$ OTU counts were collapsed by shared taxonomy at all taxonomic levels. Modelling was carried out for each level individually in the same manner as for OTUs. These analyses were repeated within the subset of individuals who had not used antibiotics.

\section{Interventional study replication}

To further assess the possible causal link between exposure to PPIs and the observed taxonomic changes in TwinsUK, we re-analysed data from a previously published crossover study. Methods for this study have been described. ${ }^{31}$ In brief, 12 healthy adult volunteers not exposed to antibiotics within the previous 12 months each took $40 \mathrm{mg}$ of omeprazole twice daily for 4-8 weeks, and donated stool samples before and after the PPI course. Bacterial DNA was extracted from all samples and the V4 region of the 16S rRNA gene was amplified using a primer set identical to that used in the TwinsUK study. As for the TwinsUK cohort, the Greengenes database was used for final taxonomic assignments. To best compare data, we assessed the taxonomic changes within the samples from immediately before and immediately after 4 weeks of omeprazole. We analysed taxa that were significantly associated with PPI in TwinsUK and present in the majority of individual specimens $(>50 \%)$ in the intervention study. We assessed the magnitude and directions of within-individual changes using rank-sum tests (when the distribution of data was not normal) or paired t tests. Taxonomies assigned as 'Other' against the Greengenes reference were not included as they were not comparable between sets.

\section{RESULTS}

\section{PPI use in the TwinsUK cohort}

Intermittent data on self-reported PPI usage and GI health over a $\sim 10$ year time span was available for 1827 individuals, comprising $374 \mathrm{DZ}$ twin pairs, $410 \mathrm{MZ}$ twin pairs and 259 singletons; $90 \%$ were female, with an average age of 62 years. Within this set, $892(49 \%)$ had reported some form of GI indication for PPIs, $229(12 \%)$ had been prescribed PPIs at some point (only 24 having used PPIs without any GI indication), and 704 (39\%) had reported neither PPI prescription nor GI indication.

\section{PPI use is associated with age, BMI, frailty, and diet}

A number of covariates were selected. These included: age, diet as quantified using the first five PCs from FFQs, BMI, and frailty. The association of these with PPI use was assessed in the subset of individuals with complete covariate data $(n=1200$, 175 having use PPIs) (figure 1). PPI users were significantly older $\left(\mathrm{p}<10^{-6}\right)$, frailer $\left(\mathrm{p}<10^{-15}\right)$, and had higher BMI $(p=0.002)$. They were also found to be significantly lower scoring on FFQ PC2 $(p=0.0003)$, a dietary component related to high alcohol intake. ${ }^{35}$

\section{Significantly lower diversity in the gut microbiome of PPI users}

There was significantly lower $(\mathrm{p}<0.05)$ diversity in the gut microbiota of PPI users compared to those not using PPIs with all diversity indices (figure 2). There was no significant difference, with any diversity metric, between the individuals with GI indications compared to those without.

The observed negative association between PPI use and $\alpha$ diversity did not withstand adjustment for family and twin structure, BMI, age, frailty and GI indication, in the 1200 individuals with complete data (see online supplementary table S2).

\section{PPI use is associated with specific taxonomic abundances}

Modelling of OTU abundances against PPI use identified 22 OTUs with significantly lower abundance in PPI users; all were assigned to the phylum Firmicutes. There were 32 OTUs positively associated with PPI use, 20 from the order Bacteroidales and seven assigned to the Streptococcus genus. The strongest association was with a Bifidobacterium OTU ( $\left(\mathrm{q}<10^{-4}, \beta=0.45\right)$, followed by a Streptococcus assigned OTU ( $\left(\mathrm{q}<10^{-4}, \beta=0.44\right)$ (see online supplementary table S3).

To identify specific taxonomic relationships, modelling was repeated against OTU abundances collapsed by shared taxonomic assignment at various depths of classification (see online supplementary table S4). A summary of significant associations is shown in figure 3 .

Seven collapsed species were negatively associated with PPI use and were all assigned to Erysipelotrichales or Clostridiales (except from one Cyanobacteria). At the level of genera, nine were found to be negatively associated with PPI use, which were largely Firmicutes, with members of the family Erysipelotrichaceae being the most significantly decreased. Five families were negatively associated with PPI use, most strongly, Lachnospiraceae $(\mathrm{q}=0.004, \beta=-0.35)$ and Ruminococcaceae $(\mathrm{q}<0.0007, \beta=-0.26)$.

There were 24 species positively associated with PPI use. These belonged to the phyla Actinobacteria, Bacteroidetes, Firmicutes (particularly Lactobacillaceae and Clostridiales) and Proteobacteria. The largest increases observed with PPI use were the species Rothia mucilaginosa $\left(\mathrm{q}<10^{-6}, \beta=0.51\right)$ and Streptococcus anginosus $\left(\mathrm{q}<10^{-6}, \beta=0.48\right)$. We observed 24 genera that were positively associated with PPI use. The most significantly increased were Rothia $\left(\mathrm{q}<10^{-5}, \beta=0.45\right)$ and Streptococcus $\left(\mathrm{q}<10^{-6}, \beta=0.47\right)$. Ten families were significantly positively associated with PPI use, the most significant being Streptococcaceae $\left(\mathrm{q}<10^{-6}, \beta=0.46\right)$ and Micrococcaceae $\left(\mathrm{q}<10^{-5}, \beta=0.46\right)$.

\section{Taxonomic associations with PPI use are independent of antibiotic use}

Self-reported oral antibiotic usage data were available for 1 month before faecal sample collection for 1039 of the 1827 individuals. Antibiotic use was significantly associated with PPI use within this set $\left(\chi^{2}(1, \mathrm{~N}=1309)=8.88, \mathrm{p}<0.002\right)$, where $16 \%$ of PPI users had used antibiotics compared to only $8 \%$ of individuals who had not used PPIs. To ensure this enrichment was not influencing the observed associations, modelling analyses were repeated within a subset of 705 individuals that had reported no antibiotic use and had complete covariate data (see online supplementary table S5).

At all levels of analysis, from OTU to phylum, results reflected those of the wider set. The number of significant associations was reduced because of the smaller sample size, but the majority of associations were retained, particularly the strongest positive associations with Streptococcaceae and other Lactobacillales, and the negative associations observed with the class Clostridia. These results show that the observed microbiome associations with PPI use are independent of increased antibiotic utilisation.

\section{Significant associations between discordant twin pairs}

The influence of PPIs on the microbiota of $70 \mathrm{MZ}$ twins discordant for PPI use was investigated to control for shared environmental and genetic effects (see online supplementary methods). 


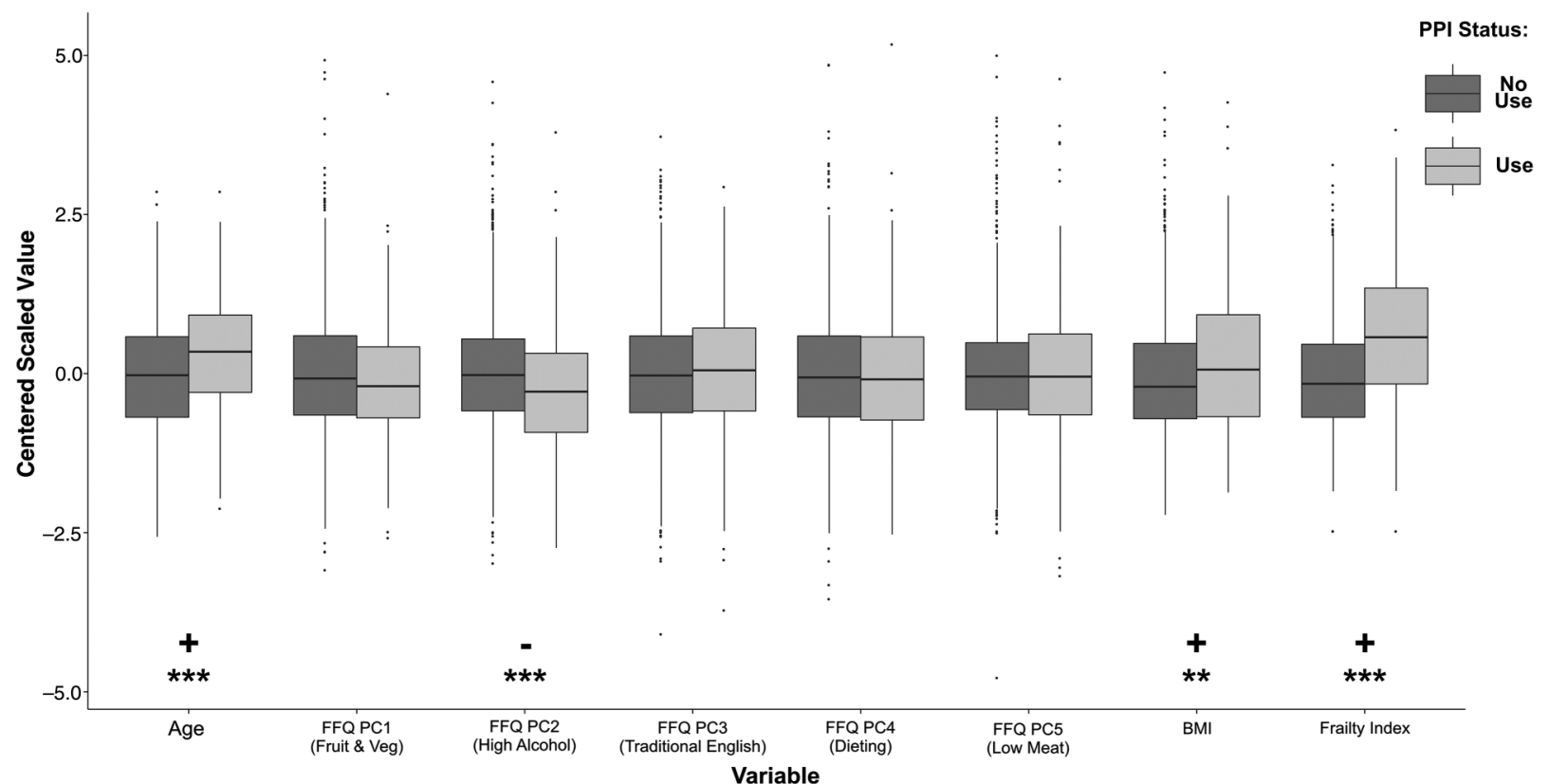

Figure 1 Distributions of covariates included for analysis, compared between proton pump inhibitor (PPI) users and non-users. Wilcoxon rank sum tests were carried out to compare the distribution of covariates in the modelling analysis. All variables were on a different scale so were centred and scaled before plotting. PPI users were older, frailer, had higher body mass index (BMI) and lower scores on the high alcohol food frequency questionnaire (FFQ) principle component (PC). Significant differences are indicated where ${ }^{* *} p<0.001$ and ${ }^{* *} p<0.01$.

No significant differences in the abundances of any OTU, species or genera were observed between discordant MZs. The Streptococcaceae family had a significantly higher abundance in PPI users within discordant twins $(\mathrm{q}=0.04, \times 2.9$ higher $)$, as did its parent order Lactobacillales $(q=0.02, \times 2.6$ higher) (figure 4$)$.

At higher taxonomic levels, significant changes were observed between twins for the classes Actinobacteria $(q=0.03, \times 1.3$ higher in PPI users), Bacilli $(q=0.03, \times 1.9$ higher $)$, and 4 C0D-2 ( $q=0.03, \times 0.3$ lower), and the phyla Actinobacteria $(q=0.04, \times 1.1$ higher), Cyanobacteria $(q=0.04, \times 0.33$ lower $)$, and Verrucomicrobia $(q=0.04, \times 0.4$ lower $)$.

\section{PPI microbiota associations replicate in an interventional study}

From the 96 collapsed taxonomies significant in the TwinsUK set, 63 were found in at least $50 \%$ of the interventional set and considered for replication (see online supplementary table S4). Within these, seven were significantly associated with PPI use in the intervention, all increasing in abundance after 4 weeks of PPI use. These were unassigned species belonging to the genera Streptococcus and Granulicatella, the Granulicatella genus, and the families Carnobacteriaceae, Streptococcaceae, Burkholderiaceae, and Corynebacteriaceae. All belonged to the order Lactobacillales except Corynebacteriaceae and Burkholderiaceae, which are from the orders Actinomycetales and Burkholderiales. Within this size-limited intervention the strongest taxa associations, particularly Lactobacilli, appear to be driven by PPI use.

\section{PPI use associated with a higher abundance of pharyngeal bacteria in the gut}

Body site of origin of the altered bacteria was investigated to shed light on the mechanism driving observed associations. Data from the human microbiome project (HMP) was used as a reference to determine body site preferences of bacterial families (see online supplementary methods and supplementary table S6). It is worth noting that individual species within each family may be commensals at different and multiple sites. Here we simply aimed to determine overall if families were more frequently identified at particular body sites.

Within the five families found to associate negatively with PPI use in TwinsUK, four were more common in the gut, with two also being found in abundance in the mouth/throat. The exception was Cyanobacteria, which was not biased towards any site in the HMP data. All 10 families positively associated with PPI use (including four replicated in the interventional study) displayed site preference. The six strongest, most significantly associated families were enriched in the mouth/throat with one also in abundance in the skin/nose, one was only enriched at the skin/nose sites, and two were most commonly found at vaginal sites. The only family most common to the gut and with increased abundance with PPI use was Burkholderiaceae. Overall, families with significantly reduced abundance with PPI use were more often found in the gut in the HMP data; while families with significantly higher abundance with PPI use were more often found in the mouth/throat, skin/nose or vaginal sites (likely a result of the large number of Lactobacillaceae commensals found here). ${ }^{23}$

To determine if this trend applied to all families, including those not significantly associated with PPI use, coefficients of association of each family with each site in the HMP data were correlated against families' associations with PPI use in the TwinsUK data. There was a non-significant negative correlation between the association with PPI use and with the gut $(\rho=$ $-0.23, p=0.07$ ), and a non-significant positive correlation with vaginal coefficients $(\rho=0.2, p=0.12)$. However, significant positive correlations were observed between the association with PPI use and the association with the mouth/throat $(\rho=0.38$, $p=0.0019)$ and the skin/nose $(\rho=0.36, p=0.003)$ sites.

\section{DISCUSSION}

We have profiled the effects of PPI use on the gut microbiome in by far the largest study to date, and considered a number of 

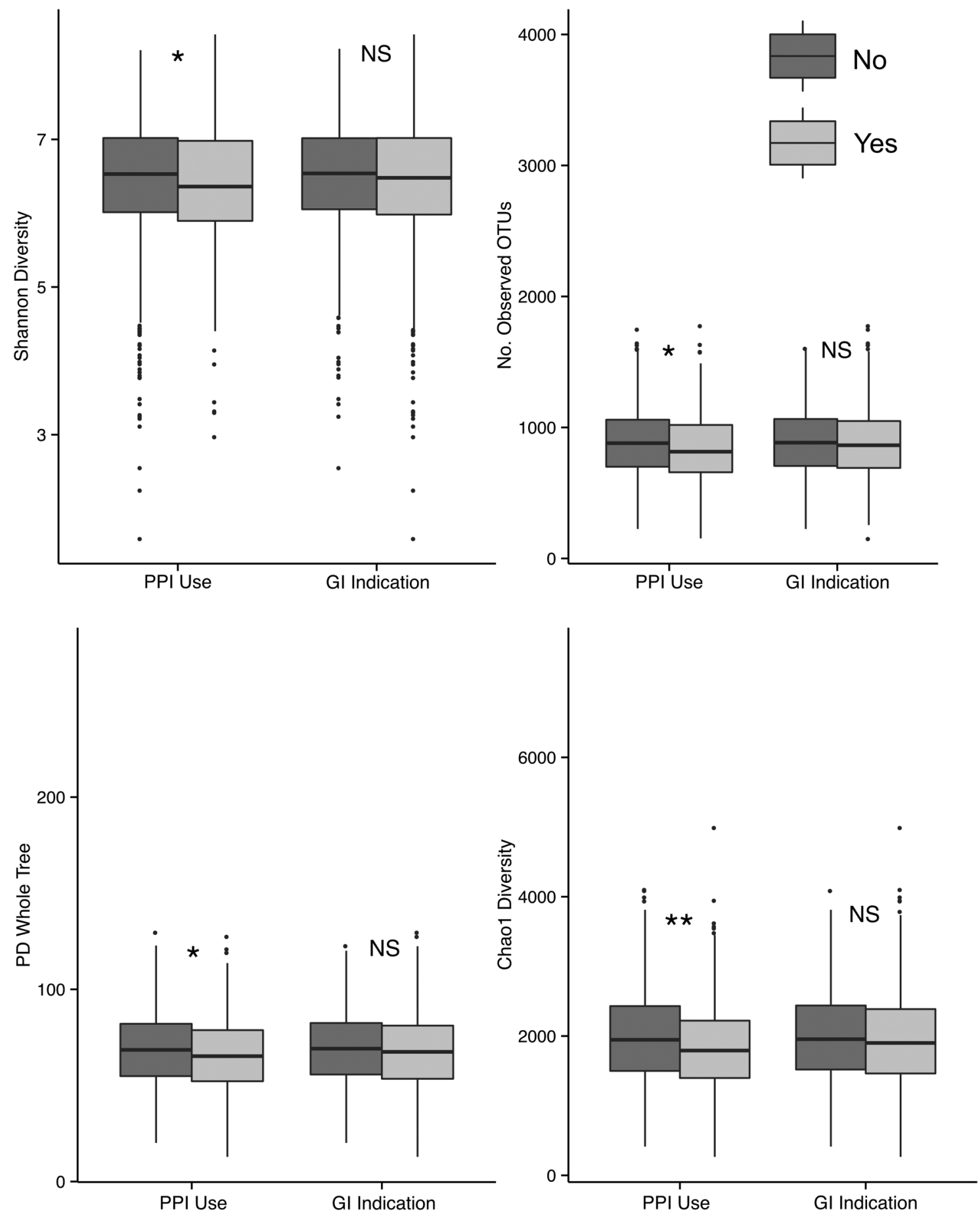

Figure 2 Comparison of $\alpha$ diversity in proton pump inhibitor (PPI) users and non-users, and in individuals with and without GI indications. Four metrics of $\alpha$ diversity were calculated on rarefied samples and one-tailed Wilcoxon rank sum tests carried out to test for significantly lower diversity with PPI use, or with GI indication. Significantly lower diversity was observed for all metrics in PPI users versus non-users (*); no difference was found splitting by GI indication (NS).

possible confounders including host genetics. We have demonstrated that PPI use is associated with an altered composition of the gut microbiota, and a moderately lower diversity. In all three analyses, the large observational study, between discordant twins, and the interventional replication, PPI use was associated with increases in the Lactobacillales order, and in particular the family Streptococcaceae. Further, we show these effects could result from downward movement of upper tract commensals.

We observed a significant reduction in microbial diversity with PPIs. However, it was a small difference and became non- significant after adjusting for covariates. This may be due to confounding effects and/or reduced power of the smaller sample. Variables we found to associate with PPIs, such as BMI, frailty, and antibiotic use, are also known to reduce diversity. ${ }^{24} 2640$ Therefore, it is likely that these factors are partly responsible for the observed lower diversity in PPI users; such confounders were also not accounted for in previous observations of decreased diversity with PPI use. ${ }^{32}$ This is further supported by a number of studies where no major changes in diversity have been observed. ${ }^{28} 30$ 
Phylum

Class

Order

Family

Genus
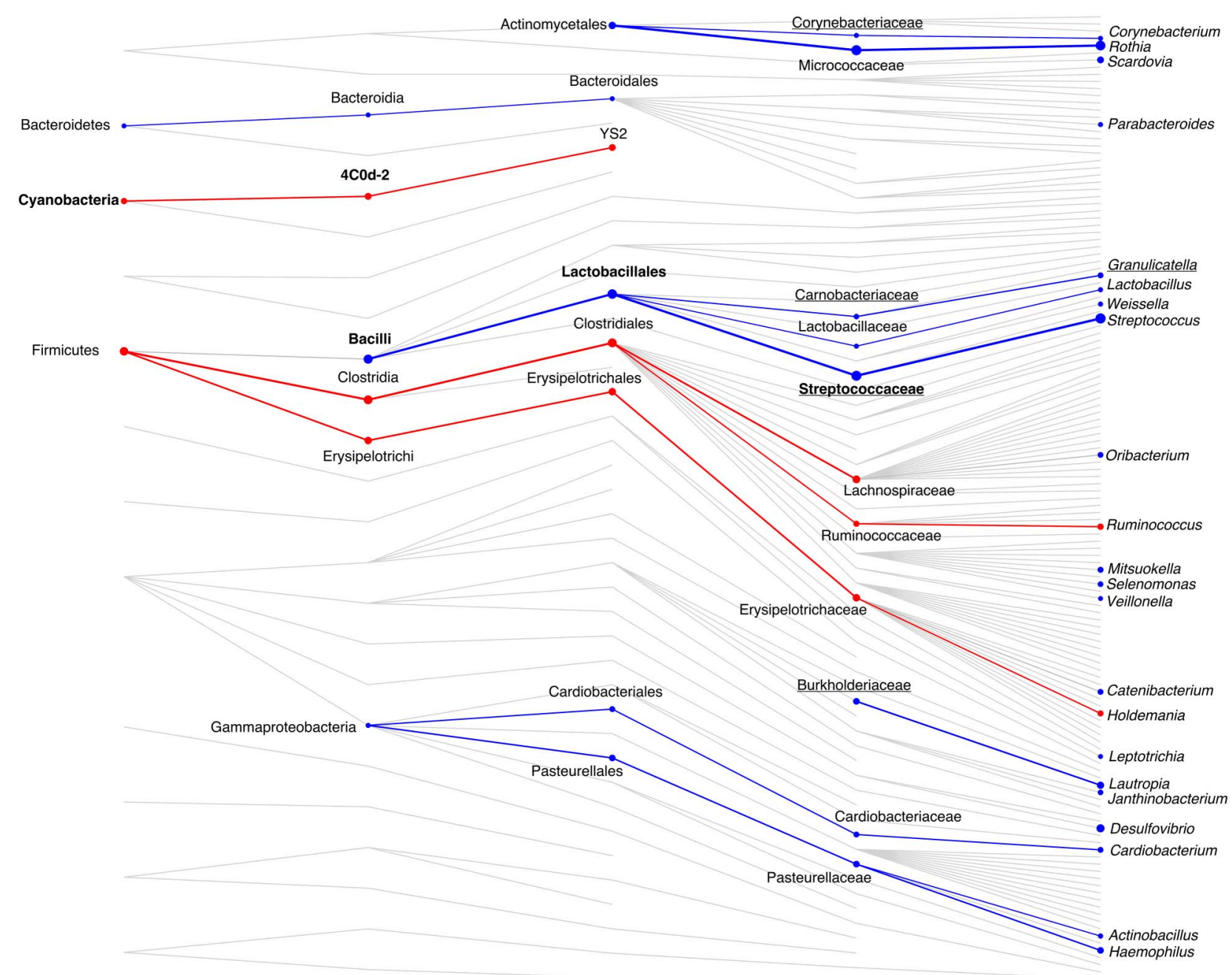

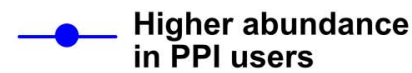

in PPI users

\section{Lower abundance}

in PPI users

Figure 3 Summary of taxonomic associations with proton pump inhibitor (PPI) use. Shown are all collapsed groups used in taxonomic association analyses that had complete taxonomic assignment (not including collapsed species). Connecting lines highlight the taxonomic relationships between groups (not considering genetic relatedness). Taxa significantly associated with PPI use are highlighted with circles, larger circles representing a larger absolute coefficient of association. Association analyses were carried out at each taxonomic level independently. Taxa at higher abundance with PPI use are shown in blue and at lower abundance in red. Lines connecting taxa of similar association are also coloured and weighted by the average coefficient between the taxa. Names are shown for significant results only. Those in bold retained significance between 70 discordant monozygotic (MZ) twins, and underlined taxa replicated in analysis of interventional study data.

There was a clear association between the composition of the microbiome and PPI utilisation. Collapsing by taxonomic assignment revealed the lineage specificity of these associations, in particular to those containing Streptococcaceae and other Lactobacilli. A number of these associations have been identified in smaller studies. For example, 8 weeks of PPI use was found to increase the abundance of Actinomycetales and Lactobacillales in the oesophagus of 34 patients suffering from heartburn. ${ }^{29}$ An increase in Streptococcus was also observed with PPI use in a case-control study of 116 children, ${ }^{30}$ and previous analyses of the intervention study data utilised within this study also identified similar increases in Streptococcaceae and Micrococcaceae. ${ }^{31}$ These studies also identified changes not present in our study, for example, increases in Gemellales, Enterococacceae, and Staphylocacceae. ${ }^{29} 31$
We found families with higher abundance with PPI use to be frequent commensals of the oral, throat, nasal, and skin communities. We hypothesise that under normal circumstances gastric acid acts as a barrier to progression down the GI tract for pharyngeal commensals and environmental bacteria, which are not well adapted to low $\mathrm{pH}$. Treatment with PPIs removes this barrier allowing colonisation by these bacteria further along the GI tract, eventually translating to the detected increased abundance in faecal samples. As observations are based on relative abundances, the observed lower abundance of gut commensals likely reflects the increase in other taxa, rather than a reduction in absolute levels.

PPIs may also act on specific bacterial taxa directly. Previous evidence suggests that they may have antimicrobial action against Helicobacter pylori. ${ }^{41}$ Also, at least one Streptococcus species is known to have P-type ATPase transporters belonging 


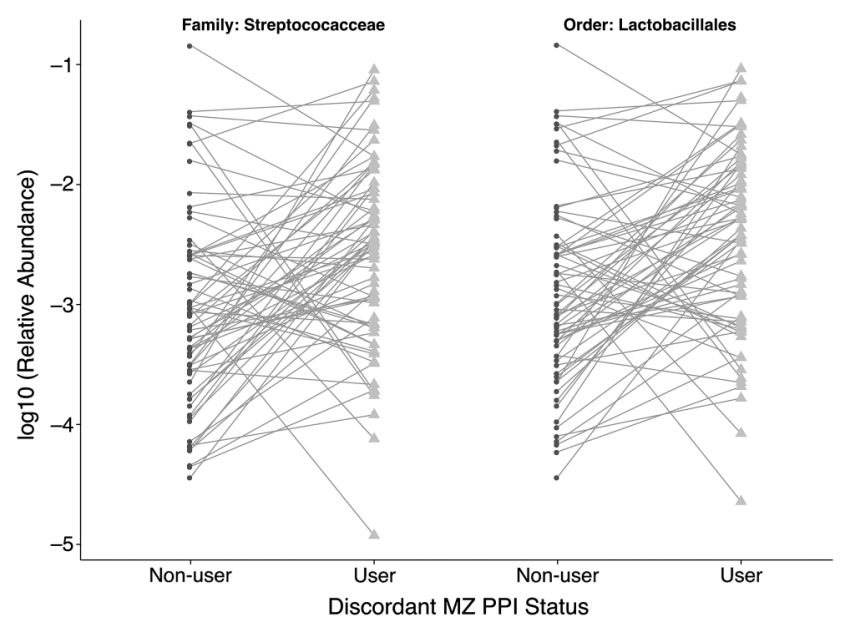

Figure 4 Paired plots of the relative abundances of Streptococcaceae and Lactobacillales within monozygotic (MZ) twins discordant for proton pump inhibitor (PPI) use. These were the only collapsed family and order traits found to be significantly different in discordant MZ twin pairs, both higher in abundance in PPI users.

to the same enzyme family as the human $\mathrm{H}+/ \mathrm{K}+$ ATPase targeted by PPIs. ${ }^{43}$ Bacterial targets for direct PPI interactions could drive species-specific compositional changes.

There are limitations to the study. The TwinsUK data are observational, although our key findings were confirmed between twin pairs and replicated in data from a small prospective controlled trial. PPI use and GI indication were self-reported and over a wide timespan from faecal sampling. However, misclassification of exposures should only serve to reduce the strength of observed associations. We have also omitted duration of PPI use as accurate data were not available, which should be considered in future investigations as it may influence the strength of microbiome associations. However, the lack of duration data would tend to dilute our associations as short and long-term users are classified together. Similarly, we did not consider the effects of withdrawal of PPI treatment, which may be important given that dysbioses resulting from antibiotic use can have long lasting effects. ${ }^{40}$

Antibiotic use was not scored for all individuals within this study; we have also not considered the effects of particular classes of antibiotics, dosage and duration of courses, or antibiotic use before the previous month. However, the robustness of our observations within the subset of individuals who had not used antibiotics shows that they are independent of the effects of recent antibiotic exposure. This study was also limited to faecal sampling. While the observations in the gut are robust, they offer no insight into the distribution of these bacteria along the GI tract. Sampling of multiple sites combined with culture experiments would determine the distribution of living bacteria, and the influence of upper tract community composition on subsequent changes in the gut. In vitro studies will also be required to elucidate whether our observations are driven by $\mathrm{pH}$ changes, direct drug interactions, or a combination of both. This will be particularly important to determine if these effects occur with other classes of acid-suppressing medication.

The associations reported here are of clinical importance. $C$ difficile affects nearly half a million people in the USA annually, ${ }^{44}$ and is known to capitalise on alterations to the normal gut microbiota. $^{27}$ The increased risk of enteric infection with PPI use may similarly be mediated through changes to the GI microbiome. It has been shown that a high abundance of Streptococcus in the gut predisposes mice to $C$ difficile colonisation, while
Lachnospiraceae are protective. ${ }^{45}$ On this basis, we observed taxonomic changes that would be expected to promote $C$ difficile infection. Further investigations into the microbiome-mediated determinants of $C$ difficile infection will be important to understand how to mitigate the risk associated with PPI use.

A further consequence for consideration is the potential for the GI tract to become a reservoir for potential pathogens at alternate body sites. A significant increased risk of community-acquired pneumonia has been observed with PPIs (relative risk 1.98 for users vs ex-users), ${ }^{18}$ and has been observed specifically for Streptococcus derived pneumonia. ${ }^{46}$ There is speculative evidence of bacterial exchanges between the gastric and lung fluids, ${ }^{30} 47$ and depletion of the gut microbiota reduces immune mediated resilience to pneumococcal pneumonia in mice. ${ }^{48}$ PPI use has also been shown to increase the risk of spontaneous bacterial peritonitis and overall bacterial infection in patients with cirrhosis and ascites, ${ }^{19}$ suggesting PPI use may pose a higher risk to individuals already susceptible to infection and other complications; for example, the elderly and the more frail or more obese individuals, whom our study indicates are more likely to be prescribed PPIs.

The described associations between PPI use and the gut microbiome warrant further research to better understand the driving mechanisms and their consequences, and are a further reason to reduce unnecessary prescribing.

Acknowledgements We thank Dr Frances Williams for her advice in preparation of the manuscript.

Contributors Statistical analyses within TwinsUK were carried out by MAJ. M-EM collated and parsed PPI and GI indication data. MAJ authored the manuscript with the help of M-EM. DEF and JAA carried out replication analysis in the interventional data set. ACP, JLS and DW carried out extraction and sequencing of DNA from samples. JKG carried out pre-processing and OTU picking from sequencing data. CJS conceived the study idea, advised on analysis and coordinated the project. REL, JTB and TDS coordinated the collection and analysis of the microbiota data. All authors contributed to and revised the manuscript.

Funding The TwinsUK microbiota project was funded the National Institutes of Health (NIH) RO1 DK093595, DP2 OD007444. TwinsUK received funding from the Wellcome Trust; European Community's Seventh Framework Programme (FP7/ 2007-2013), the National Institute for Health Research (NIHR)-funded BioResource, Clinical Research Facility and Biomedical Research Centre based at Guy's and St Thomas' NHS Foundation Trust in partnership with King's College London. CJS is funded under a grant from the Chronic Disease Research Foundation (CDRF). DEF was funded by the National Center for Advancing Translational Sciences (NIH KL2 TR000081).

Competing interests None declared.

Ethics approval Ethical approval for the HATs and microbiota studies within TwinsUK were provided by the NRES Committee London-Westminster.

Provenance and peer review Not commissioned; externally peer reviewed. Data sharing statement All data are available for request from TwinsUK.

Open Access This is an Open Access article distributed in accordance with the terms of the Creative Commons Attribution (CC BY 4.0) license, which permits others to distribute, remix, adapt and build upon this work, for commercial use, provided the original work is properly cited. See: http://creativecommons.org/ licenses/by/4.0/

\section{REFERENCES}

1 Katelaris PH. Proton pump inhibitors. Med J Aust 1998;169:208-11.

2 Bardou M, Toubouti Y, Benhaberou-Brun D, et al. Meta-analysis: proton-pump inhibition in high-risk patients with acute peptic ulcer bleeding. Aliment Pharmacol Ther 2005:21:677-86.

3 Sharma VK, Leontadis GI, Howden CW. Meta-analysis of randomized controlled trials comparing standard clinical doses of omeprazole and lansoprazole in erosive oesophagitis. Aliment Pharmacol Ther 2001;15:227-31.

4 DeVault KR, Castell DO. Updated guidelines for the diagnosis and treatment of gastroesophageal reflux disease. Am J Gastroenterol 2005;100:190-200.

5 Yachimski PS, Farrell EA, Hunt DP, et al. Proton pump inhibitors for prophylaxis of nosocomial upper gastrointestinal tract bleeding: effect of standardized guidelines on prescribing practice. Arch Intern Med 2010;170:779-83. 
6 Singh G, Triadafilopoulos G. Appropriate choice of proton pump inhibitor therapy in the prevention and management of NSAID-related gastrointestinal damage. Int $J$ Clin Pract 2005;59:1210-17.

7 Jung R, MacLaren R. Proton-pump inhibitors for stress ulcer prophylaxis in critically ill patients. Ann Pharmacother 2002;36:1929-37.

8 National Institute for Health and Clinical Excellence. NSAIDs_-prescribing issues. 2015. http://cks.nice.org.uk/nsaids-prescribing-issues (accessed 5 Jul 2015).

9 IMS Health Top-Line Market Data. 2014. http://www.imshealth.com/portal/site/ imshealth/menuitem.18c196991f79283fddc0ddc01ad8c22a/? vgnextoid=6521e590cb4dc310VgnVCM100000a48d2ca2RCRD\&vgnextfmt=default (accessed 6 Jul 2015).

10 Akram F, Huang $Y$, Lim V, et al. Proton pump inhibitors: are we still prescribing them without valid indications? Australas Med J 2014;7:465-70.

11 Eid SM, Boueiz A, Paranji S, et al. Patterns and predictors of proton pump inhibitor overuse among academic and non-academic hospitalists. Intern Med 2010;49:2561-8.

12 Heidelbaugh JJ, Kim AH, Chang R, et al. Overutilization of proton-pump inhibitors: what the clinician needs to know. Therap Adv Gastroenterol 2012;5:219-32.

13 Fitton A, Wiseman L. Pantoprazole. Drugs 1996;51:460-82.

14 Wilde MI, McTavish D. Omeprazole. Drugs 1994;48:91-132.

15 Langtry HD, Wilde MI. Lansoprazole. Drugs 1997;54:473-500.

16 Valuck RJ, Ruscin JM. A case-control study on adverse effects: $\mathrm{H} 2$ blocker or proton pump inhibitor use and risk of vitamin B12 deficiency in older adults. J Clin Epidemiol 2004;57:422-8.

17 Leonard J, Marshall JK, Moayyedi P. Systematic review of the risk of enteric infection in patients taking acid suppression. Am J Gastroenterol 2007; 102:2047-56.

18 Laheij RJF, Sturkenboom MCJM, Hassing, et al. Risk of community-acquired pneumonia and use of gastric acid-suppressive drugs. JAMA 2004;292:1955-60.

$19 \mathrm{Xu} \mathrm{HB}$, Wang HD, Li CH, et al. Proton pump inhibitor use and risk of spontaneous bacterial peritonitis in cirrhotic patients: a systematic review and meta-analysis. Genet Mol Res 2015;14:7490-501.

20 Janarthanan S, Ditah I, Adler DG, et al. Clostridium difficile-associated diarrhea and proton pump inhibitor therapy: a meta-analysis. Am J Gastroenterol 2012;107:1001-10.

21 Kwok CS, Arthur AK, Anibueze Cl, et al. Risk of Clostridium difficile infection with acid suppressing drugs and antibiotics: meta-analysis. Am J Gastroenterol 2012;107:1011-19.

22 Faust K, Sathirapongsasuti JF, Izard J, et al. Microbial co-occurrence relationships in the Human Microbiome. PLoS Comput Biol 2012;8:e1002606.

23 The Human Microbiome Project Consortium. Structure, function and diversity of the healthy human microbiome. Nature 2012;486:207-14.

24 Turnbaugh PJ, Hamady M, Yatsunenko T, et al. A core gut microbiome in obese and lean twins. Nature 2009;457:480-4.

25 Willing BP, Dicksved J, Halfvarson J, et al. A pyrosequencing study in twins shows that gastrointestinal microbial profiles vary with inflammatory bowel disease phenotypes. Gastroenterology 2010;139:1844-54.e1.

26 Claesson MJ, Jeffery IB, Conde $\mathrm{S}$, et al. Gut microbiota composition correlates with diet and health in the elderly. Nature 2012;488:178-84.

27 Owens RC, Donskey CJ, Gaynes RP, et al. Antimicrobial-associated risk factors for Clostridium difficile infection. Clin Infect Dis 2008;46(Suppl 1):S19-31.

28 Tsuda A, Suda W, Morita $H$, et al. Influence of proton-pump inhibitors on the luminal microbiota in the gastrointestinal tract. Clin Trans/ Gastroenterol 2015;6:e89.
29 Amir I, Konikoff FM, Oppenheim M, et al. Gastric microbiota is altered in oesophagitis and Barrett's oesophagus and further modified by proton pump inhibitors. Environ Microbiol 2014;16:2905-14.

30 Rosen R, Hu L, Amirault J, et al. 16S community profiling identifies proton pump inhibitor related differences in gastric, lung, and oropharyngeal microflora. J Pediatr 2015;166:917-23.

31 Freedberg DE, Toussaint NC, Chen SP, et al. Proton pump inhibitors alter specific taxa in the human gastrointestinal microbiome: a crossover trial. Gastroenterology 2015;149:883-885.e9.

32 Seto $C T$, Jeraldo $P$, Orenstein $R$, et al. Prolonged use of a proton pump inhibitor reduces microbial diversity: implications for Clostridium difficile susceptibility. Microbiome 2014:2:42.

33 Goodrich JK, Waters JL, Poole AC, et al. Human genetics shape the gut microbiome. Cell 2014;159:789-99.

34 Caporaso JG, Kuczynski J, Stombaugh J, et al. QIIME allows analysis of high-throughput community sequencing data. Nat Methods 2010;7:335-6.

35 Moayyeri A, Hammond CJ, Valdes AM, et al. Cohort profile: TwinsUK and healthy ageing twin study. Int J Epidemiol 2013;42:76-85.

36 Teucher B, Skinner J, Skidmore PML, et al. Dietary patterns and heritability of food choice in a UK female twin cohort. Twin Res Hum Genet 2007;10:734-48.

37 Searle SD, Mitnitski A, Gahbauer EA, et al. A standard procedure for creating a frailty index. BMC Geriatr 2008;8:24.

38 Bates $\mathrm{D}$, Mächler M, Bolker BM, et al. Fitting linear mixed-effects models using Ime4. ArXIV e-print; Press J Stat Softw 2015;67:1-48.

39 Dabney A, Storey JD. qvalue: Q-value estimation for false discovery rate control. R package version 2.2.0 2015 https://www.bioconductor.org/packages/3.3/bioc/ $\mathrm{html} / \mathrm{qvalue}$.html

40 Jakobsson HE, Jernberg C, Andersson AF, et al. Short-term antibiotic treatment has differing long- term impacts on the human throat and gut microbiome. PLOS ONE 2010;5:e9836.

41 Hirai M, Asuma T, Ito $S$, et al. A proton pump inhibitor, E3810, has antibacterial activity through binding to Helicobacter pylori. J Gastroenterol 1995;30:461-4.

42 Nagata K, Satoh $\mathrm{H}$, Iwahi T, et al. Potent inhibitory action of the gastric proton pump inhibitor lansoprazole against urease activity of Helicobacter pylori: unique action selective for H. pylori cells. Antimicrob Agents Chemother 1993;37:769-74.

43 Ajdić D, McShan WM, McLaughlin RE, et al. Genome sequence of Streptococcus mutans UA159, a cariogenic dental pathogen. Proc Natl Acad Sci USA 2002;99:14434-9.

44 Longo DL, Leffler DA, Lamont JT. Clostridium difficile infection. N Engl J Med 2015;372:1539-48.

45 Schubert AM, Sinani H, Schloss PD. Antibiotic-induced alterations of the murine gut microbiota and subsequent effects on colonization resistance against Clostridium difficile. MBio 2015;6:e00974.

46 de Jager CPC, Wever PC, Gemen EFA, et al. Proton pump inhibitor therapy predisposes to community-acquired Streptococcus pneumoniae pneumonia. Aliment Pharmacol Ther 2012;36:941-9.

47 Dumoulin G. Aspiration of gastric bacteria in antacid-treated patients: a frequent cause of postoperative colonisation of the airway. Lancet 1982;319:242-5.

48 Schuijt T, Lankelma J, Scicluna BP, et al. The gut microbiota plays a protective role in the host defence against pneumococcal pneumonia. Gut 2016;65: 575-83. 